\title{
Application of Mathematics in civil Engineering
}

\author{
Mayur Jain \\ Assistant Professor \\ Department of Civil Engineering SATI, Vidisha (M.P.)
}

\begin{abstract}
Application of mathematics in various streams of engineering is well known by everyone. In civil engineering there is several applications of mathematics tools to analyse or simplify various problems like finite element analysis of structure, particle study in liquid, air and in solid state, Determination of various Sectional properties etc. It is impossible to analysis of fluid mechanics, rigid mechanics and solid mechanics without use of mathematical tools of trigonometry, calculus, algebra and geometry.
\end{abstract}

Keywords: Application of mathematics in Fluid Flow, FEM, Mechanics.

\section{INTRODUCTION}

Civil engineering is a profession that applies mathematical and physical science principles to design and develop structures and methods to utilize materials and forces of nature for the benefit of humanity. Civil engineers create buildings, industrial plants and transportation infrastructure. Today, civil engineering is often combined with environmental engineering as cities and countries place more focus on sustainable building and protecting infrastructure from damage caused by natural disasters. In fluid mechanics in the analysis of type of motion of fluid particle, type of flow, energy equation, continuity equation, velocity potential, stream function etc. all can only be defined by application of mathematics by using eulerian and langrangian approach of particle analysis, curl of function, vector calculus etc. In structural analysis finite element method is very useful for analysis of multistorey structures, design of rigid pavements for roads, analysis of particle under various kind of forces like seismic force, wind force etc. Various software's of analysing of civil structures are based on FEM method of analysis like staad pro, etabs, ansys, sap etc. Many of important theorems of engineering mechanics like Lami's theorem, Varignon's theorem, euler's theorem etc. are the key tools for analysing of particles in rest or as well as in motion under the action of external forces. Also in determining of various properties like area, volume, centroid, moment of inertia, radius of gyration, slenderness ratio etc. of different sections like rectangle, triangle, circle and compound section mathematics had played vital role.

\section{How Mathematics USES IN Civil ENGinEeRing}

Civil engineering coursework involves the application of mathematical principles and skills to real world problems. Classes such as

Structural analysis examine structures like trusses, beams and frames, and Concepts like

Virtual work, energy methods and influence lines. Mechanics of solids includes topics like internal forces and deformation in solids, stresses and deflections in beams, and column theory and analysis. Courses in fluid mechanics involve study of the properties of fluids, fluid dynamics and dynamic similitude to analyze the flow of compressible and incompressible fluids in closed conduits.

All of these classes apply the principles and skills learned in the prerequisite mathematics courses.

\section{SOME OF THE MATHEMATICAL TOOLS THAT ARE USED IN CIVIL ENGINEERING}
a. TRIGONOMETRY
b. CALCULUS
c. ALGEBRA
d. PROBABILITY \& STATISTICS
e. GEOMETRY
f. LINEAR ALGEBRA AND
g. DIFFERENTIAL EQUATIONS

\section{A. Trigonometry}

The branch of mathematics dealing with the relations of the Sides and angles of the triangle and with the relevant function of any angles. Civil engineers use trigonometry to calculate angles and elevation when a building is to be plan. Civil engineers use Trigonometry often when surveying a structure. Surveying deals with land elevations as well as the various angles of structures. 


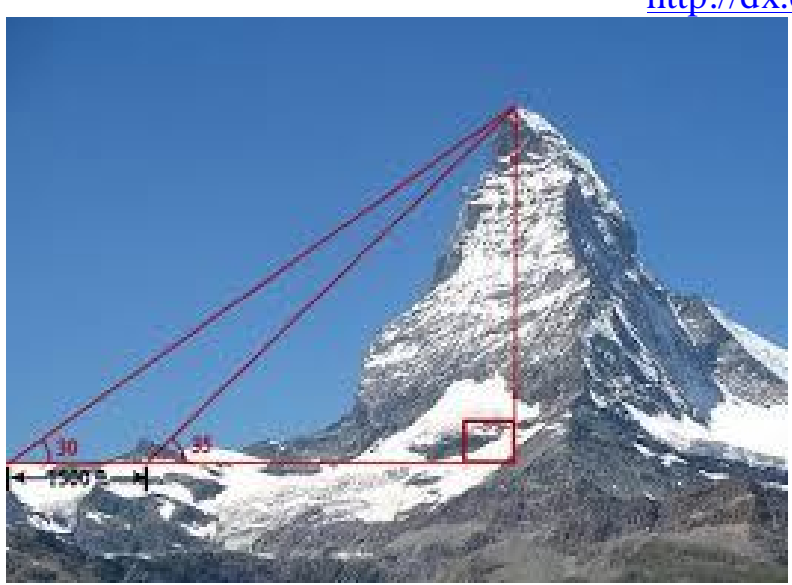

B. Geometry

* Of all the Engineering disciplines, Civil Engineering uses Geometry the most. Geometry means "to measure the earth" and clearly Civil Engineers involved in surveying are doing precisely that.

* More generally, Geometry involves the analysis of shapes and the relationships among them. Civil Engineers must know how to design and assemble shapes to construct buildings, dams, bridges, tunnels, highway systems, etc. The geometry of those shapes determines their functionality.

* For the shapes used, a Civil Engineer must understand and know how to compute such quantities as lengths, areas, volumes, centroids, moments of inertia, and curvatures, and must be able to determine the spatial relationship among these shapes.

* Advanced software breaks up objects into elemental pieces (e.g., triangles, pyramids, cubes) to determine the stresses and strains within them.

* Descriptive Geometry helps Civil Engineers visualize structures and objects and engage in their design and analysis.

* Fractal Geometry (the newest branch of Geometry) is used by Civil Engineers to analyze such entities as the friction between objects, the clumping of materials, and the porosity of soils, all of which involve geometric patterns that repeat on an ever decreasing scale.

\section{Probability and Statistics}

Measuring and analysing risk is a key skill in engineering, and most civil engineering programs require students take courses in probability and statistics to develop the skills and knowledge to quantify risk and safety in their designs.

Courses in statistics cover topics like frequency interpretation of probability, probability theory, discrete probability and combinatory, distribution and density functions, and sampling theory.

These courses also introduce the use of computer software to perform statistical analysis on the kind of large data sets that are involved in engineering problems like primavera etc.

\section{Calculus}

Calculus is the study of the rate of change in functions. Most civil engineering programs require calculus. Calculus is a prerequisite for most civil engineering courses. It consist

* Derivatives and integrals of functions in one dimension. These classes also cover topics like velocity, acceleration and optimization.

* Calculus may also be required in a civil engineering program, deals with functions in two and threed dimensions, and includes topics like surface and volume integrals, and partial derivatives.

\section{E. Linear Algebra and Differential Equations}

Most civil engineering programs require courses in linear algebra and differential equations. These courses are prerequisites for upper-division civil engineering courses.

* Linear algebra, or matrix algebra, involves the study of solutions to systems of equations, determinants and vector spaces, including function spaces, linear transformations and quadratic forms.

* Differential equations, is a more advanced mathematics course required by some civil engineering programs. It includes topics like first-order differential equations, undetermined coefficients and systems of linear differential equations, with a focus on applications to science and engineering.

\section{CONCLUSION}

ENGINEERING + MATHS $=$ EVERYTHING

ENGINEERING - MATHS $=$ NOTHING 